\title{
Assessment of Insulin Injection Technique Among Diabetic Patients in Makkah Region in Saudi Arabia
}

\author{
Ghadeer A. Alhazmi ${ }^{1}$, Rafal N. Balubaid ${ }^{2}$, Samaher Sajiny ${ }^{3}$, Rufaydah Alsabbah ${ }^{3}$ \\ 1. Medicine, Umm Al-Qura University, Makkah, SAU 2. Internal Medicine, Umm Al-Qura University, Makkah, SAU 3. \\ Pediatrics, Umm Al-Qura University, Makkah, SAU
}

Corresponding author: Rafal N. Balubaid, rafalbalubaid@gmail.com

\begin{abstract}
Introduction: Diabetes mellitus (DM) is defined as a chronic metabolic disorder characterized by persistent high blood glucose. Proper insulin injection is indispensable to achieve adequate control of DM and prevent complications. Therefore, this study aims to assess the knowledge of DM patients about their insulin injection techniques.
\end{abstract}

Materials and Methods: A questionnaire-based and cross-sectional study was carried out from January to March 2020 at three locations in Makkah, Saudi Arabia. The inclusion criteria of research subjects included patients diagnosed with type-1 or type-2 DM and using insulin pen injection. The questionnaire included demographic data including age, gender, nationality, educational level, and chronic disease as well as specific questions about insulin injection technique.

Results: Four hundred and thirty-seven DM patients participated in the study. The most prevalent age group was between 20 and 60 years old (69.1\%). The vast majority of patients were females (64.1\%), Saudi nationals (92.9\%), and residents of Makkah city (70\%). There were roughly equal numbers of patients with type- 1 and type- 2 DM ( $47 \%$ and $53 \%$, respectively). With regard to complications of DM, $19.5 \%$ of patients had previous acidosis, and $16.5 \%$ of patients were admitted to hospital for various complications. Injectionrelated complications were reported by almost half of the patients (49.9). The knowledge of insulin injection practices was examined among DM patients according to different variables. Only the frequency of administration exhibited a significant difference in the practices toward proper insulin injection technique $(p=0.049)$; patients who administered insulin injection three times daily had the best practices toward insulin injection when compared to other patients.

Conclusions: Our study showed that the practices toward insulin use among the surveyed DM patients in Makkah region were not satisfactory. Poor insulin injection technique is an important modifiable risk factor for uncontrolled blood glucose levels. More awareness campaigns and better counseling initiatives are crucial to guarantee steady insulin levels among DM patients.

Received 08/18/2020

Review began 08/18/2020

Review ended 09/24/2020

Published 09/27/2020

() Copyright 2020

Alhazmi et al. This is an open access article distributed under the terms of the Creative Commons Attribution License CC-BY 4.0., which permits unrestricted use, distribution, and reproduction in any medium, provided the original author and source are credited.
Categories: Internal Medicine, Quality Improvement, Public Health

Keywords: diabetes mellitus type 2, insulin injection, diabetes mellitus type 1

\section{Introduction}

Diabetes mellitus (DM) is defined as a chronic metabolic disorder characterized by persistent high blood glucose. It is one of the rapidly evolving disorders globally, and some countries have reached epidemic levels [1]. Specifically, Saudi Arabia is one of the leading ten countries worldwide with a high prevalence rate close to $24 \%[2]$.

Proper insulin injection and regular blood tests are indispensable to achieve an adequate control of DM [1,35]. Frid et al. demonstrated global improper insulin injection techniques by DM patients from 42 countries [6]. Contemporary reports have demonstrated a positive correlation between insulin injection misuse and poor glucose control $[7,8]$.

Improper insulin injection technique is a common phenomenon. It can lead to glycemic variability and subsequently compromised short- and long-term complications [5,9]. Such aftermaths include injection siterelated pain, bruising, allergy, infection, and lipodystrophy $[10,11]$.

In Saudi Arabia, a recently published systematic review by Alanazi et al. depicted poor public awareness about DM and its risk factors as well as complications [12]. This poor awareness about DM raises a serious concern about the proper insulin injection techniques among DM patients. Therefore, the aim of this study is to assess the knowledge of DM patients about their insulin injection techniques. 


\section{Materials And Methods}

A cross-sectional study using a questionnaire-based survey was carried out at three locations in Makkah, Saudi Arabia. These locations included: (1) primary healthcare centers, (2) the Department of Endocrinology at King Faisal Hospital Center, and (3) the Diabetic Center at Hira General Hospital. The study was conducted from January to March 2020. The inclusion criteria included patients diagnosed with type-1 or type-2 DM using insulin pen injection. Patients using insulin syringes were excluded. The research protocol was approved by the Ethics Committee at Umm Al-Qura University, Makkah, Saudi Arabia (identification number: HAPO-02-K-012-2018-08-08-263).

The questionnaire of study was prepared based on previous literature with modifications [13-15]. The questionnaire was conducted in an interview-based setting. There were two parts of the questionnaire with a total of 40 questions. The first part of questionnaire included questions about demographics, such as age, gender, nationality, educational level, and chronic diseases. The second part of questionnaire included questions relating specifically to DM, such as characteristics of DM, complications of DM, practices of insulin injection, insulin pen injection techniques, and sources of information about insulin injection.

The questionnaire was piloted on 20 research participants to gauge the validity and interpretation of questions. The questionnaire was found to be comprehensible, and thus no further changes were made.

With regard to questions about technical practices of insulin pen injection among the surveyed patients, each question which was answered correctly was given one point (a maximum score of nine points per patient).

Data were analyzed using the Statistical Package for the Social Sciences (SPSS, IBM Corp., Armonk, New York). Data were presented as numbers and frequencies. Two-tailed one-way analysis of variance (ANOVA) test was used to compare the average score of insulin injection techniques according to various sociodemographic and clinical variables. A p value less than 0.05 was regarded statistically significant.

\section{Results}

Four hundred and thirty-seven DM patients participated in the study. Table 1 shows the patients' sociodemographics. Age was subcategorized into three age groups, and the most prevalent age group was between 20 and 60 years old (69.1\%). The vast majority of patients were females (64.1\%), Saudi nationals (92.9\%), and residents of Makkah city (70\%). Close to half of the patients had a bachelor's degree (49.9\%) and other coexisting comorbidities (53.5\%). 


\section{Cureus}

\begin{tabular}{|lll|}
\hline Variable & & $n(\%)$ \\
\hline Age & $<20$ years old & $40(9.2)$ \\
& 20 to 60 years old & $302(69.1)$ \\
Gender & 60 years old & $95(21.7)$ \\
& Male & $157(35.9)$ \\
\hline Nationality & Female & $280(64.1)$ \\
& Saudi & $406(92.9)$ \\
& Non-Saudi & $31(7.1)$ \\
Educational level & Illiterate & $36(8.2)$ \\
& Primary & $40(9.2)$ \\
& Secondary & $78(17.8)$ \\
& Intermediate & $30(6.9)$ \\
\hline Existing comorbidities & Bachelor & $218(49.9)$ \\
& Postgraduate & $35(8)$ \\
& Yes & $253(57.9)$ \\
& No & $184(42.1)$ \\
\hline
\end{tabular}

\section{TABLE 1: The surveyed patients' sociodemographics.}

There were roughly equal numbers of patients with type- 1 and type- 2 DM ( $47 \%$ and $53 \%$, respectively). With regard to complications of DM, $19.5 \%$ of patients had previous acidosis, $17.6 \%$ of patients had previous seizures, and $16.5 \%$ of patients were admitted to hospital for various complications.

Table 2 depicts information about the use of insulin by patients. Roughly $57 \%$ had been using insulin for more than five years. Insulin was self-administered in around three quarters of patients (72.1\%) and mostly once (30.7\%) or twice (30\%) daily.

\begin{tabular}{|c|c|c|}
\hline \multicolumn{2}{|l|}{ Question } & $\mathrm{n}(\%)$ \\
\hline \multirow{3}{*}{ When did you start using insulin? } & Less than two years & $84(19.2)$ \\
\hline & Two to five years & $104(23.8)$ \\
\hline & More than five years & $249(57)$ \\
\hline \multirow{5}{*}{ What is the frequency of insulin administration? } & Once daily & $134(30.7)$ \\
\hline & Twice daily & $131(30)$ \\
\hline & Three times daily & $108(24.7)$ \\
\hline & Four times daily & $55(12.6)$ \\
\hline & Five times daily & $9(2.1)$ \\
\hline \multirow{2}{*}{ Who gives you insulin injection? } & Someone & $122(27.9)$ \\
\hline & Myself & 315 (72.1) \\
\hline
\end{tabular}

TABLE 2: Information about the surveyed patients' use of insulin pen. 


\section{Cureus}

correctly had stored insulin inside fridge door, washed hands before injection, checked insulin expiration date, and used alcohol swab to rub the site of injection, respectively. Injection-related complications were reported by almost half of the patients $(49.9 \%)$.

\begin{tabular}{|c|c|c|}
\hline \multicolumn{2}{|l|}{ Question } & n (\%) \\
\hline \multirow{3}{*}{ Where do you store insulin? } & Fridge door* & $239(54.7)$ \\
\hline & Inside the fridge & $164(37.5)$ \\
\hline & Room temperature & $34(7.8)$ \\
\hline \multirow{3}{*}{ Do you wash your hands before injection? } & Yes $^{*}$ & $179(41)$ \\
\hline & No & $104(23.8)$ \\
\hline & Sometimes & $154(35.2)$ \\
\hline \multirow{3}{*}{ Do you check the expiry date? } & Yes $^{*}$ & 331 (75.7) \\
\hline & No & $60(13.7)$ \\
\hline & Sometimes & 46 (10.5) \\
\hline \multirow{2}{*}{ Do you check the type of insulin that you should take? } & Yes & $397(90.8)$ \\
\hline & No & $40(9.2)$ \\
\hline \multirow{5}{*}{ What is the most common area that you inject? } & Upper arm & $151(34.6)$ \\
\hline & Thigh & $148(33.9)$ \\
\hline & Abdomen & $132(30.2)$ \\
\hline & Calf muscle & $5(1.1)$ \\
\hline & Buttocks & $1(0.2)$ \\
\hline \multirow{3}{*}{ Do you change the site of injection every time? } & Yes $^{*}$ & $241(55.1)$ \\
\hline & No & $44(10.1)$ \\
\hline & Sometimes & $152(34.8)$ \\
\hline \multirow{4}{*}{ What do you use to sterilize the injection site? } & I do not rub injection site & $128(29.3)$ \\
\hline & Water & $4(0.9)$ \\
\hline & Medical swab* & $276(63.2)$ \\
\hline & Tissue & $29(6.6)$ \\
\hline \multirow{3}{*}{ Do you wait till the site of injection dries from alcohol? } & Yes $^{*}$ & $144(33)$ \\
\hline & No & $187(42.8)$ \\
\hline & Sometimes & $106(24.3)$ \\
\hline \multirow{3}{*}{ Do you inject the recommended dose? } & Yes $^{*}$ & $338(77.3)$ \\
\hline & No & $51(11.7)$ \\
\hline & Sometimes & $48(11)$ \\
\hline \multirow{3}{*}{ Do you notice any complications after using the needle? } & Yes & $48(11)$ \\
\hline & No & $219(50.1)$ \\
\hline & Sometimes & $170(38.9)$ \\
\hline
\end{tabular}

TABLE 3: The surveyed patients' practices of insulin pen injection.

* Correct answers for the questions. 


\section{Cureus}

Table 4 displays the technical practices of insulin injection. Only $65.7 \%, 9.6 \%$, and $50.3 \%$ of patients correctly reported inserting the needle perpendicularly, discarding the needles in special baskets, and raising the skin at the site of injection, respectively. Around $65 \%$ of patients believed injecting insulin correctly.

About $58.7 \%$ had injection-related complications such as bleeding and bruising, while $35.3 \%$ of the patients reported lipohypertrophy.

\begin{tabular}{|c|c|c|}
\hline \multicolumn{2}{|l|}{ Question } & n (\%) \\
\hline \multirow{3}{*}{ What is the angle that you use to inject insulin? } & Perpendicular (90 degrees) & $287(65.7)$ \\
\hline & Inclined (45 degrees) & 89 (20.4) \\
\hline & Flat & $61(14)$ \\
\hline \multirow{3}{*}{ Do you leave the needle in your body after injecting insulin? } & Yes & $245(56.1)$ \\
\hline & No & $161(36.8)$ \\
\hline & I do not know & $31(7.1)$ \\
\hline \multirow{4}{*}{ How long do you keep it? } & Five to ten seconds & $257(58.8)$ \\
\hline & Ten to 60 seconds & $29(6.6)$ \\
\hline & More than 60 seconds & $1(0.2)$ \\
\hline & I do not keep it & 150 (34.3) \\
\hline \multirow{3}{*}{ Do you rotate the injection site after the injection? } & Yes & $148(33.9)$ \\
\hline & No & $166(38)$ \\
\hline & Sometimes & $123(28.1)$ \\
\hline \multirow{3}{*}{ How many times do you reuse the needle? } & One to five times & 119 (27.2) \\
\hline & More than five times & $44(10.1)$ \\
\hline & I do not reuse it & $274(62.7)$ \\
\hline \multirow{3}{*}{ Where do you throw the needle? } & Special basket & $42(9.6)$ \\
\hline & Bin & $356(81.5)$ \\
\hline & Both & $39(8.9)$ \\
\hline \multirow{3}{*}{ Do you return the cover to the needle after use? } & Yes & 399 (91.3) \\
\hline & No & $14(3.2)$ \\
\hline & Sometimes & $24(5.5)$ \\
\hline \multirow{3}{*}{ Are you making skin fold? } & Yes & $220(50.3)$ \\
\hline & No & $133(30.4)$ \\
\hline & Sometimes & 84 (19.2) \\
\hline \multirow{3}{*}{ Do you think that you inject insulin correctly? } & Yes & $283(64.8)$ \\
\hline & No & $8(1.8)$ \\
\hline & Not sure & $146(33.4)$ \\
\hline
\end{tabular}

TABLE 4: Technical practices of insulin pen injection among the surveyed patients.

More than one third of patients (37.3\%) reported reusing the needle more than one time. The reasons for reusing the needle are shown in Figure 1. 


\section{Cureus}

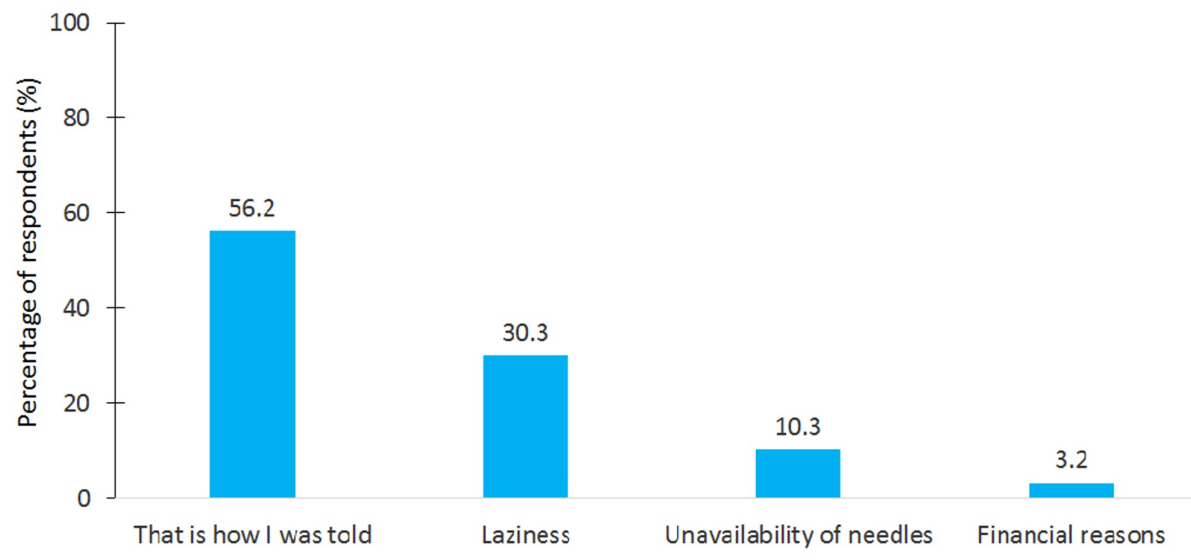

FIGURE 1: Reasons for reusing the insulin needle among the surveyed patients.

The sources of information about insulin injection are depicted in Figure 2. The vast majority of patients obtained the information from doctors $(60.2 \%)$, and only $2.1 \%$ of patients obtained them from social media.

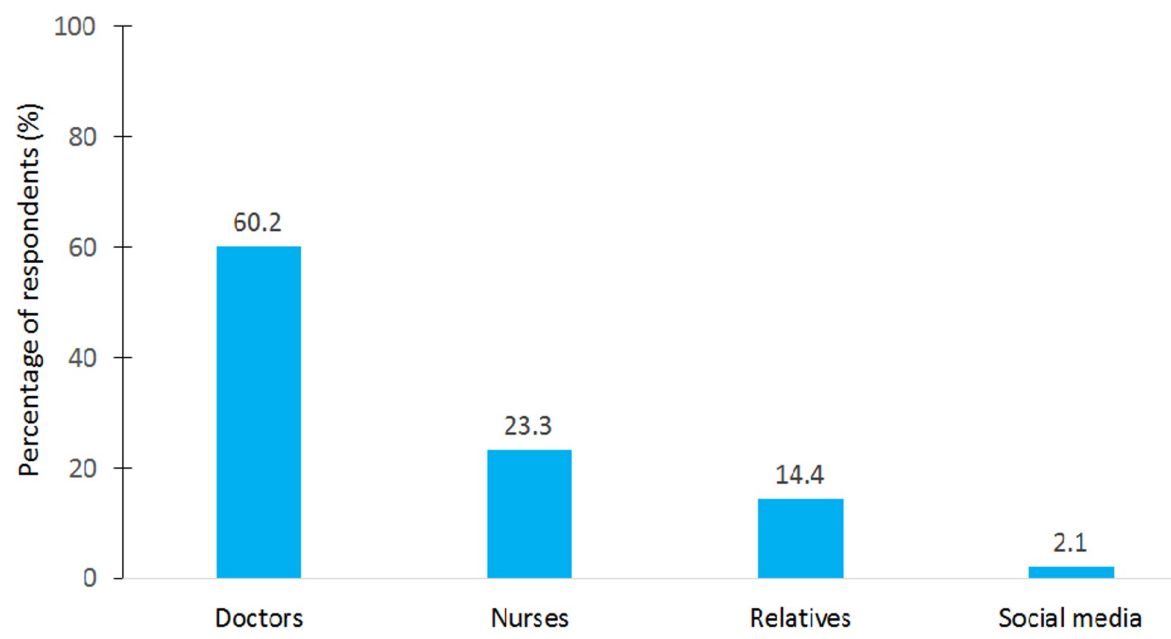

FIGURE 2: Sources of information about insulin injection among the surveyed patients.

Table 5 depicts the scores of the technical practices of insulin injection among the surveyed DM patients according to different sociodemographic and clinical variables. The mean \pm standard deviation score of the entire cohort was $5.3 \pm 1.8$. Only the frequency of administration exhibited a significant difference in practices toward insulin injection ( $p=0.049$ ); patients who administered insulin injection three times daily had the best practices toward insulin injection when compared to other patients.

\begin{tabular}{|c|c|c|c|}
\hline \multicolumn{2}{|l|}{ Variable } & Mean \pm standard deviation & $\mathrm{p}$ value \\
\hline \multirow{3}{*}{ Age } & $<20$ years old & $5.0 \pm 1.7$ & \multirow{3}{*}{0.548} \\
\hline & 20 to 60 years old & $5.3 \pm 1.9$ & \\
\hline & $>60$ years old & $5.3 \pm 1.7$ & \\
\hline \multirow{4}{*}{ Gender } & Male & $5.2 \pm 1.7$ & \multirow{4}{*}{0.353} \\
\hline & Female & $5.3 \pm 1.8$ & \\
\hline & Illiterate & $5.6 \pm 1.8$ & \\
\hline & Primary & $5.3 \pm 1.8$ & \\
\hline
\end{tabular}




\section{Cureus}

\begin{tabular}{|c|c|c|c|}
\hline \multirow{2}{*}{ Educational level } & Secondary & $5.3 \pm 1.7$ & \multirow{2}{*}{0.436} \\
\hline & Intermediate & $5.5 \pm 1.6$ & \\
\hline \multirow{4}{*}{ Type of diabetes } & Bachelor & $5.2 \pm 1.8$ & \multirow{4}{*}{0.635} \\
\hline & Postgraduate & $4.8 \pm 1.8$ & \\
\hline & Type one & $5.2 \pm 1.7$ & \\
\hline & Type two & $5.3 \pm 1.8$ & \\
\hline \multirow{3}{*}{ Duration of diabetes } & Less than five years & $5.5 \pm 1.7$ & \multirow{3}{*}{0.224} \\
\hline & Five to ten years & $5 \pm 1.9$ & \\
\hline & More than ten years & $5.2 \pm 1.7$ & \\
\hline \multirow{7}{*}{ Type of insulin } & Rapid-acting & $5.2 \pm 1.7$ & \multirow{7}{*}{0.093} \\
\hline & Long-acting & $4.1 \pm 1.6$ & \\
\hline & Mixed & $5.5 \pm 1.7$ & \\
\hline & Long-acting and mixed & $5.1 \pm 2$ & \\
\hline & Long-acting and rapid-acting & $5.4 \pm 1.8$ & \\
\hline & Rapid-acting and mixed & $5.0 \pm 1.9$ & \\
\hline & All of them & $5.5 \pm 1.6$ & \\
\hline \multirow{5}{*}{ Frequency of insulin administration } & Once daily & $5 \pm 1.8$ & \multirow{5}{*}{$0.049^{\star}$} \\
\hline & Twice daily & $5.2 \pm 1.9$ & \\
\hline & Three times daily & $5.6 \pm 1.4$ & \\
\hline & Four times daily & $5.5 \pm 1.8$ & \\
\hline & Five times daily & $4.7 \pm 1.9$ & \\
\hline \multirow{3}{*}{ Do you think that you inject insulin correctly? } & Yes & $5.3 \pm 1.9$ & \multirow{3}{*}{0.490} \\
\hline & No & $5.4 \pm 1.7$ & \\
\hline & Not sure & $5.2 \pm 1.8$ & \\
\hline \multirow{3}{*}{ Do you notice any complications after using the needle? } & Yes & $5.3 \pm 1.8$ & \multirow{3}{*}{0.961} \\
\hline & No & $5.2 \pm 1.8$ & \\
\hline & Sometimes & $5.3 \pm 1.7$ & \\
\hline
\end{tabular}

\section{TABLE 5: Scores of insulin injection practices of the surveyed patients according to various}

variables.

Two-tailed ANOVA test was used to compare the average score of insulin injection techniques according to various variables. A p value less than 0.05 was regarded statistically significant.

\section{Discussion}

$\mathrm{DM}$ is a global health disease [13]. Hence, compliance of patients with their medical treatment is crucial to reduce the incidence of complications [13]. Insulin can be used as monotherapy or in combination with other therapies to control blood glucose levels. However, the maximal benefit of insulin depends on its appropriate administration [13]. Inappropriate insulin injection technique can result in erratic levels of insulin in the body, and it is one of the major reasons leading to poor glycemic control [11]. Accordingly, the incidence of DM complications can rise significantly leading to mortality [16]. Therefore, it is crucial to understand the practices of DM patients toward their insulin injection technique [14].

Saudi Arabia ranks among the top ten countries that harbor high prevalence rates of DM [2]. In this study, we sought to assess the practices of insulin pen use among DM patients in Makkah region, Saudi Arabia. In 
this study, we sought to assess the practices of insulin pen use among DM patients in Makkah region, Saudi Arabia. We targeted DM population about their insulin injection technique.

Our study showed that roughly one fifth of the surveyed patients experienced various DM-related complications, such as acidosis, seizures, and hospital admissions. Additionally, injection-related complications were reported by almost half of the patients. Moreover, our study showed that a large proportion of the surveyed patients had unsatisfactory knowledge about insulin injection practices. Overall, these data suggest the need to enhance DM control generally and improve the insulin injection practices specifically.

Knowledge about insulin use and its technique has been evaluated in different studies. Sweidan et al. examined the level of competency of insulin pen use among 165 patients in Saudi Arabia [15]. The authors showed that nearly $75 \%$ of the surveyed participants had poor knowledge. Poudel et al. scrutinized the insulin injection practices among 43 patients in Nepal [4]. The authors revealed that there were substantial gaps concerning proper insulin injection practice. Moreover, close to $30 \%$ of patients $(n=13)$ self-reported injection-related complications. Pozzuoli et al. demonstrated suboptimal insulin injection technique among 352 Italian patients with DM [17]. The authors reported a high (43\%) prevalence of lipodystrophy among the studied subjects. A multivariate analysis revealed a positive association between occurrence of lipodystrophy and errors in insulin injection technique, such as improper rotation of needle at the site of injection and inappropriate spacing between insulin doses. Ji et al. conducted a national survey in China to gauge the daily insulin pen injection practice among 380 patients [18]. The authors demonstrated that close to one third of patients (35.3\%) had lipohypertrophy, and more than half of the patients (58.7\%) had injection-related complications such as bleeding and bruising. The authors highlighted practical errors in insulin pen injection among the surveyed DM patients.

Factors associated with reduced insulin adherence include smoking, young age, low socioeconomic status, level of scholarship, presence of other comorbidities, and polypharmacy [19]. In our study, only the frequency of administration exhibited a significant difference in practices toward insulin injection $(\mathrm{p}=$ 0.049); patients who administered insulin injection three times daily had the best practices toward insulin injection when compared to other patients. In line with an experience-based trend, we hypothesized that patients who inject insulin frequently would achieve the highest scores. The number of patients who used insulin five times and more was the smallest $(n=9)$, and this might have negatively affected the statistical analysis and masked the true outcome of an experience-based trend.

Our study mandates the need to boost the awareness of insulin injection technique among DM patients. The healthcare professionals-including physicians, nurses, and health educators-play the most central roles in this process. To that end, newly diagnosed patients with DM and requiring insulin injection should undergo one-to-one sessions about how to execute proper insulin injection. Moreover, those DM patients should be continuously counseled and examined for their insulin injection techniques during check-up visits. Lastly, educational campaigns and reliable social media platforms can provide reliable scientific materials to further strengthen the proper insulin injection practices.

The limitation of this study is that it depends mainly on patients' response to the questionnaire (recall bias) and not actual observation by healthcare professionals. As a result, the findings of this self-reported questionnaire are liable to overestimation/underestimation by the patients and may not accurately reflect the patients' actual responses. This limitation will be addressed in a forthcoming study in which patients will be observed by healthcare professionals and scored while injecting insulin.

\section{Conclusions}

Our study showed that the practices toward insulin use among the surveyed DM patients in Makkah region were not satisfactory. Poor insulin injection technique is an important modifiable risk factor for uncontrolled blood glucose levels. Improving the use of insulin injection has favorable outcomes in reducing the risk of DM complications. More awareness campaigns and better counseling initiatives are crucial to guarantee steady insulin levels among the DM patients.

\section{Additional Information \\ Disclosures}

Human subjects: Consent was obtained by all participants in this study. Ethics Committee at Umm Al-Qura University, Makkah, Saudi Arabia issued approval HAPO-02-K-012-2018-08-08-263. The research protocol was approved by the Ethics Committee at Umm Al-Qura University, Makkah, Saudi Arabia (identification number: HAPO-02-K-012-2018-08-08-263). Animal subjects: All authors have confirmed that this study did not involve animal subjects or tissue. Conflicts of interest: In compliance with the ICMJE uniform disclosure form, all authors declare the following: Payment/services info: All authors have declared that no financial support was received from any organization for the submitted work. Financial relationships: All authors have declared that they have no financial relationships at present or within the previous three years with any organizations that might have an interest in the submitted work. Other relationships: All authors 
have declared that there are no other relationships or activities that could appear to have influenced the submitted work.

\section{References}

1. Crasto W, Jarvis J, Khunti K, Davies MJ: New insulins and new insulin regimens: a review of their role in improving glycaemic control in patients with diabetes. Postgrad Med J. 2009, 85:257-267. 10.1136/pgmj.2008.067926

2. Naeem Z: Burden of diabetes mellitus in Saudi Arabia . Int J Health Sci (Qassim). 2015, 9:V-VI.

3. Mekuria AB, Gebresillassie BM, Erku DA, Haile KT, Birru EM: Knowledge and self-reported practice of insulin injection device disposal among diabetes patients in Gondar town, Ethiopia: a cross-sectional study. J Diabetes Res. 2016, 2016:1897517. 10.1155/2016/1897517

4. Poudel RS, Shrestha S, Piryani RM, Basyal B, Kaucha K, Adhikari S: Assessment of insulin injection practice among diabetes patients in a tertiary healthcare centre in Nepal: a preliminary study. J Diabetes Res. 2017, 2017:8648316. 10.1155/2017/8648316

5. Kalra S, Mithal A, Sahay R, et al.: Indian injection technique study: population characteristics and injection practices. Diabetes Ther. 2017, 8:637-657. 10.1007/s13300-017-0243-X

6. Frid AH, Hirsch LJ, Menchior AR, Morel DR, Strauss KW: Worldwide injection technique questionnaire study: injecting complications and the role of the professional. Mayo Clin Proc. 2016, 91:1224-1230. 10.1016/j.mayocp.2016.06.012

7. Tumminia A, Crimi S, Sciacca L, et al.: Efficacy of real-time continuous glucose monitoring on glycaemic control and glucose variability in type 1 diabetic patients treated with either insulin pumps or multiple insulin injection therapy: a randomized controlled crossover trial. Diabetes Metab Res Rev. 2015, 31:61-68. 10.1002/dmrr.2557

8. Snyder LL, Stafford JM, Dabelea D, et al.: Socio-economic, demographic, and clinical correlates of poor glycaemic control within insulin regimens among children with type 1 diabetes: the SEARCH for diabetes in youth study. Diabet Med. 2019, 36:1028-1036. 10.1111/dme.13983

9. Kapoor U, Ramasamy G, Selvaraj K, Sahoo JP, Kar SS: Does one-to-one demonstration with insulin pads by health-care providers improves the insulin administration techniques among diabetic patients of a tertiary care teaching hospital in South India?. Indian J Endocrinol Metab. 2016, 20:767-771. 10.4103/22308210.192904

10. Spollett G, Edelman SV, Mehner P, Walter C, Penfornis A: Improvement of insulin injection technique: examination of current issues and recommendations. Diabetes Educ. 2016, 42:379-394. $10.1177 / 0145721716648017$

11. Patil M, Sahoo J, Kamalanathan S, et al.: Assessment of insulin injection techniques among diabetes patients in a tertiary care centre. Diabetes Metab Syndr. 2017, 11:53-56. 10.1016/j.dsx.2016.09.010

12. Alanazi FK, Alotaibi JS, Paliadelis P, Alqarawi N, Alsharari A, Albagawi B: Knowledge and awareness of diabetes mellitus and its risk factors in Saudi Arabia. Saudi Med J. 2018, 39:981-989. 10.15537/smj.2018.10.22938

13. Tosun B, Cinar FI, Topcu Z, et al.: Do patients with diabetes use the insulin pen properly? . Afr Health Sci. 2019, 19:1628-1637. 10.4314/ahs.v19i1.38

14. Thomas A, Brinkkötter P, Schänzer W, Thevis M: Metabolism of human insulin after subcutaneous administration: a possible means to uncover insulin misuse. Anal Chim Acta. 2015, 897:53-61. 10.1016/j.aca.2015.09.036

15. Sweidan BA, Al Ajlouni MM, Robert AA, Alzaid AA: Competency of prefilled insulin pen usage among diabetes patients in Saudi Arabia: a cross-sectional study. Curr Diabetes Rev. 2019, 15:240-246. 10.2174/1573399814666180528081552

16. Sami W, Ansari T, Butt NS, Hamid MRA: Effect of diet on type 2 diabetes mellitus: a review . Int J Health Sci (Qassim). 2017, 11:65-71.

17. Pozzuoli GM, Laudato M, Barone M, Crisci F, Pozzuoli B: Errors in insulin treatment management and risk of lipohypertrophy. Acta Diabetol. 2018, 55:67-73. 10.1007/s00592-017-1066-y

18. Ji J, Lou Q: Insulin pen injection technique survey in patients with type 2 diabetes in mainland China in 2010. Curr Med Res Opin. 2014, 30:1087-1093. 10.1185/03007995.2014.895711

19. Alsayed KA, Ghoraba MK: Assessment of diabetic patients' adherence to insulin injections on basal-bolus regimen in diabetic care center in Saudi Arabia 2018: cross sectional survey. J Family Med Prim Care. 2019, 8:1964-1970. 10.4103/jfmpc.jfmpc_276_19 